\title{
Identification of non-coding RNAs embracing microRNA-143/145 cluster
}

\author{
Akio lio*1, Yoshihito Nakagawa², Ichiro Hirata², Tomoki Naoe ${ }^{3}$ and Yukihiro Akao 1,4
}

\begin{abstract}
In a variety of cancers, altered patterns of microRNA (miRNA) expression are reported and may affect the cell cycle and cell survival. Recent studies suggest that the expression level of miRNAs that act as tumor suppressors is frequently reduced in cancers because of chromosome deletions, epigenetical changes, aberrant transcription and disturbances in miRNA processing. miR-143 and -145, which are located approximately $1.3 \mathrm{~kb}$ from each other at chromosome 5q33, are highly expressed in several tissues, but down-regulated in most cancers. However, the mechanism of this downregulation has not been investigated in detail. Here, we show that both miRNAs were expressed well under the same control program in human tissues, but were down-regulated equally in the most of the cancer cell lines tested. Then we identified the host gene encoding both miRNAs. The transcripts of this gene were approximately $11,7.5$, and $5.5 \mathrm{~kb}$ long; and the expression of these transcripts was coordinated with that of its resident miRNAs and down-regulated in the cancer cell lines tested as well as in colorectal cancer tissue samples. These data demonstrate that the host gene can function as a primary miRNA transcript and suggest that the down-regulation of host gene expression caused the low-expression of its encoded microRNAs-143 and -145 in human cancer cell lines and in cancer tissues.
\end{abstract}

\section{Findings}

MicroRNAs (miRNAs) are tiny, endogenously expressed noncoding RNAs (18-25 nucleotides in length) that act as crucial posttranscriptional regulators of gene expression [1-3]. For several miRNAs, their participation in essential biological processes has been proved, such as in cell proliferation control, cell lineage fate decision, cell survival, tissue patterning for development, and cell metabolism [4]. Cancer is a very complex genetic disease characterized by alterations in genes encoding oncogenic and tumor-suppressor proteins [5]. Recently, it has been noted that the expression profiles of miRNAs can be used for classification, diagnosis, and prognosis of human malignancies; and the deletion or amplification of the locus encoding an miRNA in a variety of cancers has been reported. Altered patterns of miRNA expression may affect cell-cycle and survival programs and be involved in tumor initiation and progression. We previously found that microRNA-143 (miR-143) and -145 (miR-145) were down-regulated in colon cancers [6,7], gastric cancers [8], chronic lymphocytic leukemias, and B

*Correspondence: aiio@giib.or.jp

1 Department of Medical Oncology, Gifu International Institute of Biotechnology, 1-1 Naka-Fudogaoka, Kakamigahara, Gifu 504-0838, Japan Full list of author information is available at the end of the article cell lymphomas [9], and in several human cancer cell lines [7]. Several groups also reported the down-regulation of both of these miRNAs in many other types of cancers, such as bladder cancers and their cell lines $[10,11]$, cervical cancers and their cell lines [12], colorectal cancers [13-16], nasopharyngeal carcinoma [17], and prostate cancer [18]. Furthermore, such abnormal expression was found not only in malignant cells but also in cells in premalignant stages such as colon adenoma cells $[13,19]$. The introduction of the mature type of either miR-143 or -145 into colon cancer cells [6,7,20], B cell lymphoma [9], and gastric cancer cells $[8,21]$ results in a significant growth inhibition that occurs in a dose-dependent manner; and the target genes, ERK5 [22] and KRAS [20] for $m i R-143$ and IRS-1 [23] and $c-m y c$ [21] for $m i R-145$, were posttranscriptionally down-regulated. Taken together, these findings suggest that miR-143 and -145 act as tumor suppressors and provide an important clue in the study of the mechanism of tumor initiation and progression involving miRNAs.

In the present study, we identified non-coding RNAs carrying an miR-143 and -145 cluster (NCR143/145: Non-coding RNA encoding $m i R-\underline{143 / 145}$ ) and investigated the expression of NCR143/145 in all cancer cell lines tested. Importantly, the down-regulation of this host 
gene expression caused the low expression of both miRNAs in human cancer cell lines, which could lead to tumor development and progression.

\section{Expression of $\mathbf{m i R}-143$ and $\mathbf{- 1 4 5}$}

We examined the expression levels of mature miR-143 and -145 in human normal tissues by performing TaqMan microRNA assays (Fig. 1). In human normal tissues, miR-143 and -145 showed good expression in stomach, intestine, cervix, uterus, colon, and prostate (Fig. 1A, B). Whereas, in cancer cell lines, they were expressed at an extremely low level compared with that in human normal cell lines (Additional file 1 - Figure S1). Compared with their expression in corresponding normal tissues, the expression levels of both miRNAs were obviously downregulated in all cancer cell lines and cancer tissue samples tested, just as many groups had previously reported [618]. Such a similar expression pattern of them indicates
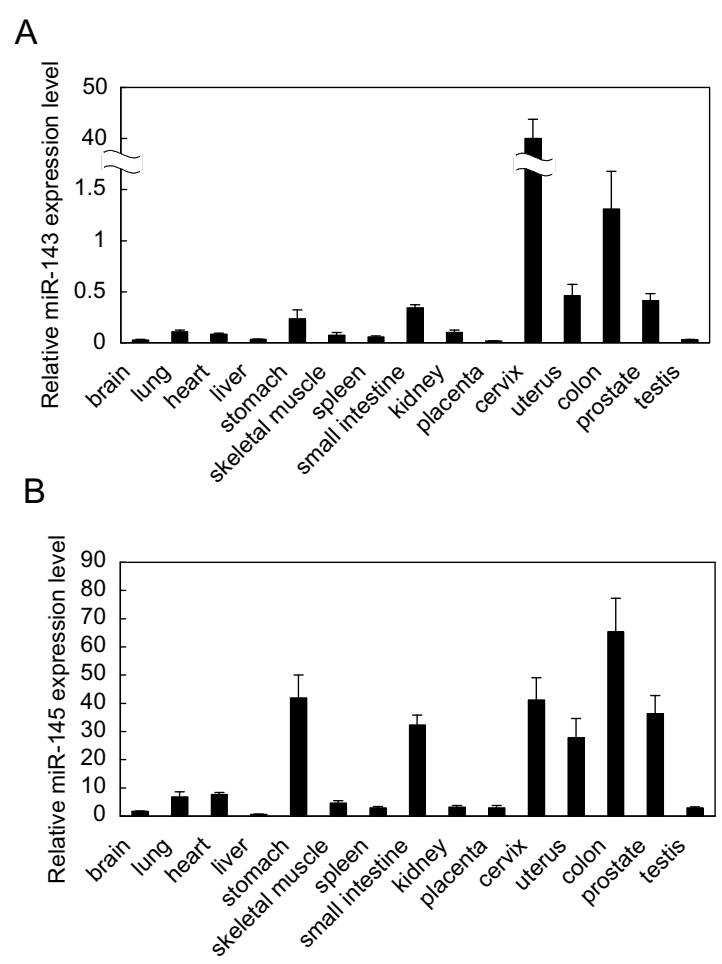

Figure 1 Real-time RT-PCR analysis of mature miR-143 and -145 expression in human normal tissues. Total RNAs from human normal tissues were purchased from Biochain (Hayward, USA) and Takara (Otsu, Japan). Expression of both miRNAs in each sample was detected by using a TaqMan microRNA reverse transcription kit and TaqMan microRNA Assay Kit (Applied Biosystems, Foster City, USA) and normalized with RNU6B. Calculation of the Ct value was done by using the second derivative maximum method, and relative quantification was analyzed by the comparative $C t(\triangle C t)$ method. All reactions were run in triplicate. Relative miR-143 (A) and -145(B) expression levels (value of $\left.2^{-\Delta C t(m i R-R N U 6 B)}\right)$ are indicated on the left axis, with error bars indicating the standard deviation for these analyses. that the expression of both miRNAs may be regulated by a similar mechanism. Additionally, the DNA loci of both miRNAs are very close to each other, within $1.3 \mathrm{~kb}$, which led us to speculate that both precursors may originate from the same primary transcript. Genomic PCR spanning this region demonstrates the fragment in most of the cancer cell lines tested $[6,7,9]$. Therefore, we decided to isolate the gene that carried both miRNAs in a cluster.

\section{Identification of non-coding RNA carrying the miR- $143 / 145$ cluster}

First, we carried out RT-PCR and inverted PCR cloning method using human placenta and uterus cDNA, and a placental cDNA library, and isolated each of the cDNA clones designated in Figure 2. The 143-145 clone was 2.2 $\mathrm{kb}$ long and detected in human tissues such as uterus, prostate, and testis by RT-PCR (data not shown). The iPCR145 clone, which encoded $m i R-145$, was $1.7 \mathrm{~kb}$ long and corresponded to the transcriptional unit for only miR-145 identified by Sachdeva [21] and $\mathrm{Xu}$ [24]. Clone 41 was 373 bp long (Fig. 2B) and amplified at a high level in human normal tissues by semi-qRT-PCR, but hardly amplified in several cancer cell lines examined (data not shown). Clone AK126481 was $3.8 \mathrm{~kb}$ long and identical to AK126481 in GeneBank, and clone AKF1-10 was $1.8 \mathrm{~kb}$ long and overlapped with AK126481. Clone 4-35 was 129 bp long and contained a part of the predicted first exon and novel second exon (Fig. 2A). At the upstream of this predicted first exon, hypothetical transcriptional start site was localized, which was shown by Fujita [25]. Also, the homolog of this gene (IE 1071) and promoter region were cloned in the mouse by Ebisuya [26] and shown to be comparatively conserved between human and mouse. This indicates that the predicted transcriptional start site near the sequence of clone 4-35 is a putative promoter region of the $m i R-143$ and -145-encoding gene.

Next, we performed Northern blot analysis to look for the transcripts that originated from the host gene encoding $m i R-143$ and -145. The large transcript (11 kb: open arrow) and 3 or 4 transcripts $(7.5,5.5$, and $1.9 \mathrm{~kb}$ : closed arrows) were detected (Fig. 3). The 11-kb transcript was hybridized with 6 probes (Fig. 2, a-f; Fig. 3), and the 1.9$\mathrm{kb}$ one was only detected by $143-145$ (Fig. 3) and iPCR145 probes (data not shown), and not detected by the 4-35, 41, AKF1-10 or AK126481 probes (data not shown). These results indicate that the host gene was firstly transcribed into the $11-\mathrm{kb}$ transcript and then processed to the mature $m i R-143$ and -145 via 7.5 and $5.5 \mathrm{~kb}$ processed variant transcripts. This gene is the non-coding RNA shown by Ebisuya to be subject to splicing [26]. Also, in human normal tissues, $m i R-145$ was consistently expressed at higher levels than $m i R-143$ (Fig. 1A, B). Apart from both miRNAs being produced from the 11-kb transcript, $m i R-145$ would also be generated from the 
A

\section{$5 q 33$}

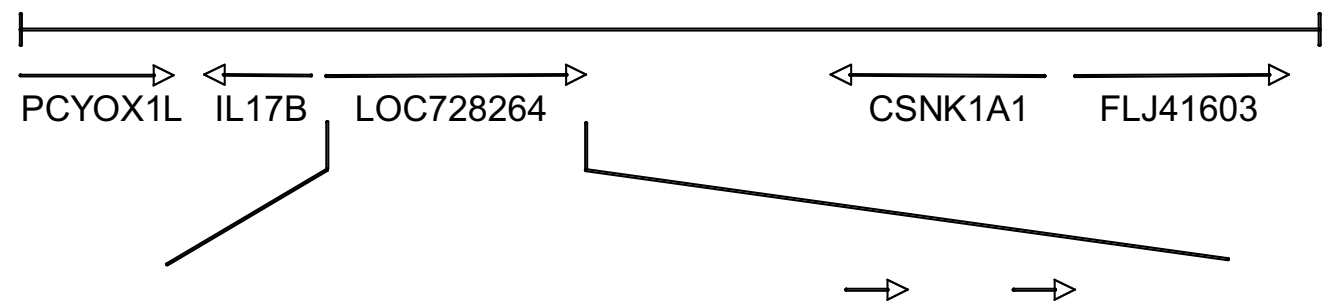

E'

miR-143 miR-145

\section{Genome}

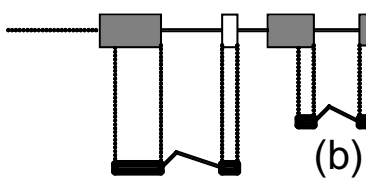

(a)

LOC3 primer

$1--1$

(A)

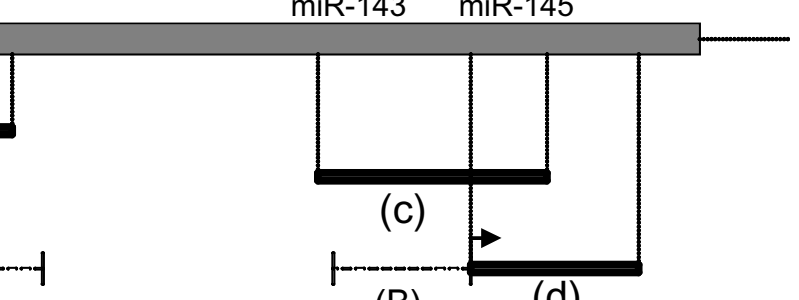

(B)

(d)

\section{(e)}

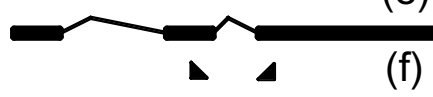

B

LOC10 primer

\begin{tabular}{|c|l|l|}
\hline & Forward primer & Reverse primer \\
\hline (a) clone4-35 & 5'-CCCAACCACTCCCCAAACAGGCTGG-3' & 5'-TTACAGCCGTTGCTCTCCTT-3' \\
\hline (b) clone41 & 5'-GCCATGCTGATGTCAGAGAA-3' & 5'-GATCCCCAGTCAGGATGAGA-3' \\
\hline (c) 143-145 & 5'-TTGGTCCTGGGTGCTCAAAT-3' & 5'-TTTCCCAAGAGTACGGCAGT-3' \\
\hline (d) iPCR145 & 5'-GAATCCCCATCTTAGCATCTAAGGGATT-3' & 5'-CTGGAAATACTGTTCTTGAGGTCATGGT-3' \\
\hline (e) AKF1-10 & 5'-GGCCATAGACCCCTCTTCTCAGTAA-3' & 5'-CTCGTGCATTTGGCTGGGGAGTGATTC-3' \\
\hline (f) AK126481 & 5'-GCTCAGTGCCAGCTGCTTAAAAAT-3' & 5'-TAGTCCTGGGCAGACCAGTTTCTATC-3' \\
\hline LOC3 & 5'-GACCAGACCCCAGGAAAGA-3' & 5'-TTACAGCCGTTGCTCTCCTT-3' \\
\hline LOC10 & 5'-AGCAAGAACTCTGGAGAAGCA-3' & 5'-GAGAGGCGTGGGTGAGAG-3' \\
\hline GAPDH(for real-time) & 5'-CCACATCGCTCAGACACCAT-3' & 5'-GCAACAATATCCACTTTACCAGAGTTAA-3' \\
\hline GAPDH( for cloning) & 5'-CCACCCATGGCAAATTCCATGGCA & 5'-TCTAGACGGCAGGTCAGGTCCACC-3' \\
\hline
\end{tabular}

Figure 2 Identification and characterization of the host gene encoding miR-143 and -145. (A) Scheme of the cytogenetic map of chromosomal region 5q33. RT-PCR cloning was performed by using the primer sets (B) that covered the predicted first exon-containing region (clone4-35;a) of LOC728264 [GeneBank:NR027180], predicted intron-spanning region (clone41;b), both miR-143 and - 145 regions (143-145;c), predicted pre-miR-145 region (iPCR145;d), AKF1-10 region (e), and AK126481 region (GeneBank;f) from human placenta and uterus CDNA, and cDNA library. The positions of neighboring genes, PCYOX1L, ILITB, CSNK1A1, and FLJ41603 are also shown as references. The real-time RT-PCR primer sets (LOC3 and 10) specific for this gene are indicated by the arrowheads. The open box (E') represents a novel exon derived from a cDNA clone (a). This exon is not shown in the NCBI database. Region "A" is a cardiac-specific enhancer reported by Cordes [29], and region "B" is p53 and/or Oct4-dependent miR-145 specific promoter reported by Sachdeva [21] and Xu [24]. 
A
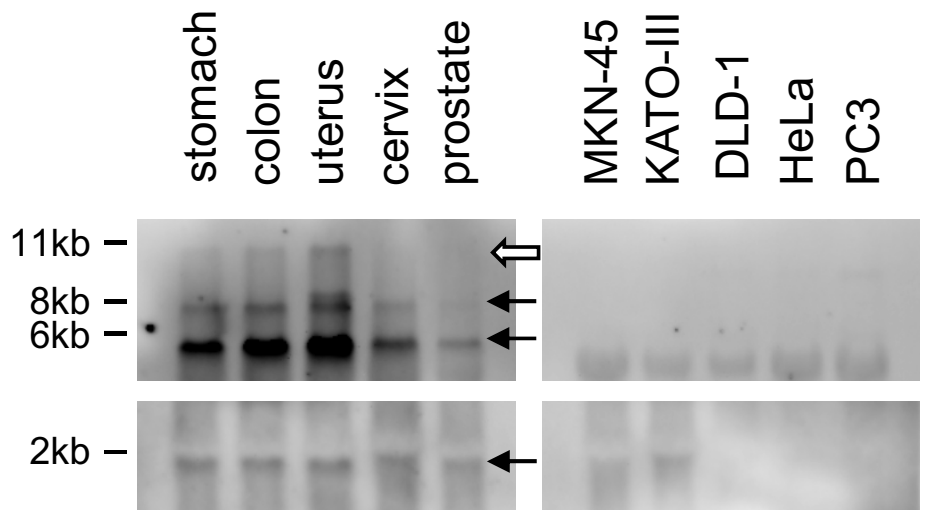

AKF1-10
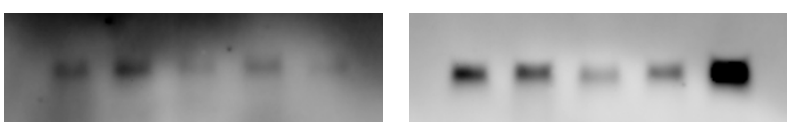

$143-145$
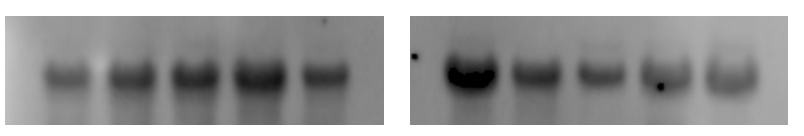

GAPDH

$28 S$

B
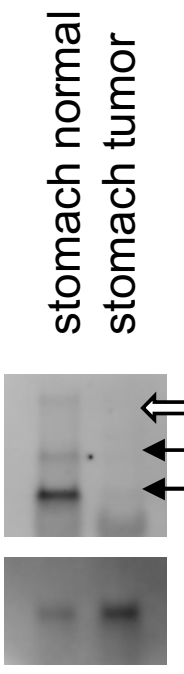

AKF1-10

\section{GAPDH}

$28 S$

Figure 3 Northern blot analysis of the host gene encoding miR-143 and -145. Total RNAs (5 $\mu \mathrm{g}$ for human normal tissues, cancer tissue, and cancer cell lines [human stomach tumor RNA was purchased from Takara]) were separated on a $1 \%(\mathrm{w} / \mathrm{v})$ formaldehyde agarose gel and then blotted onto Hybond N+ nylon membranes (Amersham Biosciences, Piscataway, USA). RNA probes were synthesized from template cDNAs by using a MAXIscript kit (Ambion, Austin, USA) incorporating Digoxigenin-11-UTP (Roche, Penzberg, Germany). Northern blots were hybridized with the AKF1-10 clone (A, upper panel; refer to Fig. 2), 143-145 clone (A, 2nd panel), GAPDH (A, 3rd panel; $\mathbf{B}$, middle panel), and $28 \mathrm{~S}$ rRNA (A and $\mathbf{B}$, under panel). The primary transcript is marked by the open arrow and other transcripts are marked by closed arrows. 
1.9-kb transcript. It is thought that this expression of miR-145 is regulated by a different mechanism dependent on p53 [21] and/or Oct4 [24]. In our preliminary experiments, the expression of host gene and its promoter activity were p53-independent in p53-mutated cancer cell lines (MKN-45 and DLD-1, data not shown). This finding of p53 independency raises the possibility that $\mathrm{p} 53$-dependent gene expression and other pathways are abrogated in p53-mutated cancer cell lines. We are currently investigated this point in our laboratory.

\section{Regulation of $N C R 143 / 145$ expression in cancers}

Most miRNAs located within protein-coding or non-coding genes are transcriptionally linked to the expression of their host genes [27]. In order to investigate the coordinated expression of the host gene identified in this study with mature miR-143 and -145, we performed real-time RT-PCR analysis by using the host gene-specific primer set shown in Fig. 2. In human normal tissues, the host gene was highly expressed, as were both miRNAs; but in the corresponding cancer cell lines, the signal was hardly detected (Fig. 4A), though the host gene and both miRNAs were highly expressed in normal human cell lines (Additional files 1 - Figure S1 \& S2). Also in human cancer tissues, the host gene was down-regulated compared with its expression in normal human tissues (Figs. 3, 4B and additional file 1 - Figure S3). As a result, the downregulation of host gene NCR143/145 expression caused low expression of both mature miRNAs in human cancer cell lines. Thus, the aberrant transcription of NCR143/ 145 could contribute to the low expression of miR-143 and -145 .

The expression level of miRNAs that act as tumor suppressors is frequently reduced in cancers because of chromosome deletions, epigenetical changes, aberrant transcription, and disturbances in miRNA processing. Michael et al. [13] reported that in colorectal cancers the decreased levels of mature miR-143 and -145 were due to reduced Dicer-processing activities. In our study, the activity and expression of Dicer and RISC proteins seemed to be intact in colorectal cancer cells, because the expression levels of miR-143 and -145 were up-regulated by stimulation with a growth inhibitor [7]. Therefore, we propose that the inadequate expression of miR-143 and 145 was due to the perturbation of transcription and/or that of the another processing enzyme, Drosha, which causes the transit from primary miRNAs to precursor ones. Recently, it was reported that $p 53$ interacts with the Drosha processing complex through association with $D D X 5$ and facilitates the processing of primary miRNAs to precursor ones [28]. That report also indicated that mutant $p 53$ interfered with these processing activities. These findings suggest that the inappropriate $p 53$-dependent modulation of miRNA biogenesis also affects the
A

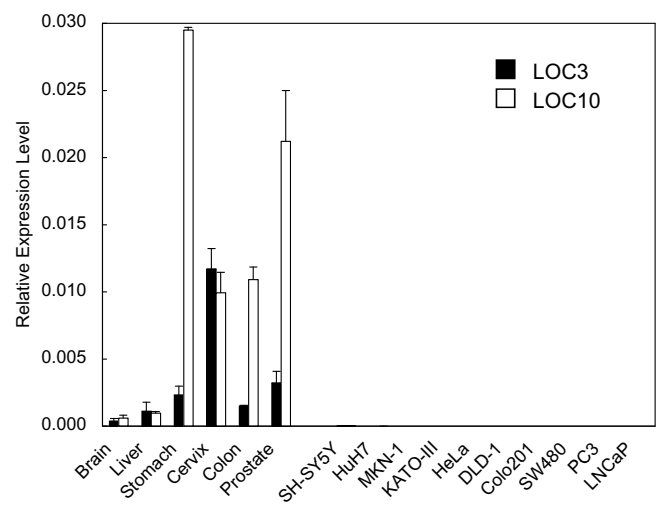

B

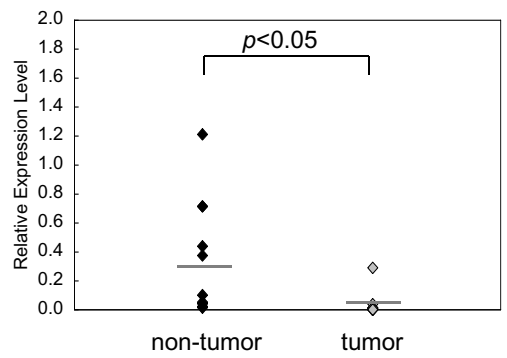

Figure 4 Real-time RT-PCR analysis of the host gene encoding miR-143 and -145. Real-time RT-PCR analysis was performed on human normal tissues and cancer cell lines $(\mathbf{A})$ and human colon adenocarcinoma samples (B) by using host gene-specific primers. Ten pairs of colon samples, including 10 human colon adenocarcinoma tissue samples and 10 matched normal colon tissue samples, were obtained from Osaka Medical College Hospital (Takatsuki, Osaka, Japan) and Fujita Health University Hospital (Toyoake, Aichi, Japan) with patients' informed consent. Collection and distribution of the samples were approved by each of the appropriate institutional review boards. The category of colon samples was confirmed by pathological analysis. Total RNA was extracted from the colon samples by using TRIzol (Invitrogen, Carlsbad, USA) according to the manufacturer's protocol. Expression levels of the host gene in each sample (LOC3 and LOC10, refer to Fig. 2) were detected by using a Superscript III reverse transcription kit (Invitrogen, Carlsbad, USA) and SYBR Premix Ex Taq (Takara, Otsu, Japan) and normalized with GAPDH. Ruled lines present the medians of samples. Data were analyzed by using Student's $t$-test. A value of $p<0.05$ was considered significant.

expression of mature miRNAs in cancer cells. But, in our study, Drosha expression and its processing activity seemed to be normal in p53-mutated MKN-45 and K562 cells (data not shown).

To exclude the possibility that the DNA of the loci and histone were epigenetically methylated, in earlier studies $[6,7,9]$ we examined by qRT-PCR the expression of both miRNAs in DLD-1, SW480 and EBV-transformed L25 cells after treatment of the cells with 5-aza-2'-deoxycytidine and tricostatin A. As a result, the levels of both miRNAs were almost unchanged by either treatment $[6,7,9]$. 
To confirm the presence of the genomic locus including both miRNAs at chromosome 5q33, we carried out genomic PCR on several cancer cell lines (Additional file 1 - figure S4) $[6,7,9]$. In HeLa, U937, and PC3 cells, one allele of the locus might have been deleted. This locus is frequently involved in chromosome copy number loss in various types of cancers including non-small cell lung cancer and gastric cancer according to the CGH database http://www.cghtmd.jp/CGHDatabase/index j.jsp. Therefore, further detailed cytogenetic study will be needed to understand the mechanism of miR-143 and -145 downregulation in many cancer cell lines.

\section{Additional material}

Additional file 1 Supplementary figures. Figure S1: Real-time RT-PCR analysis of mature $m i R-143$ and -145 expression in human cell lines.

Relative miR-143 (A) and -145 (B) expression levels are indicated on the left axis by using the comparative $\Delta C t$ method (value of $2-\Delta C t(m i R-R N U 6 B)$ ). Figure S2: Real-time RT-PCR analysis of NCR143/145 expression in human cancer cell lines and normal cell lines. The relative expression level of NCR143/145 in human cancer cell lines was compared with that in human normal cell lines (WI-38 and IMR-90) by using the LOC10 primer set (see Fig. 2). Figure S3: Real-time RT-PCR analysis of NCR143/145 expression in human stomach. The relative expression level of NCR143/145 in human stomach tumor was compared with that in normal human stomach by using the LOC10 primer set (see Fig. 2). Figure S4: Confirmation of the presence of genomic loci of $m i R-143$ and -145 at chromosome $5 q 33$ by genomic PCR. We extracted genomic DNAs from 2 cell lines and normal human oral squamous cells by using DNAzol (Invitrogen, Carlsbad, USA) and used them for PCR. The 143-145 primer set was used for genomic loci of miRs-143 and -145 (see Fig. 2B). The genomic locus of GAPDH was used as an internal control.

\section{Abbreviations}

ERK5: extracellular signal-regulated kinase 5; KRAS: v-Ki-ras2 Kirsten rat sarcoma viral oncogene homolog; IRS-1: insulin receptor substrate 1; Oct4: Octamer-4; RISC: RNA-induced silencing complex; DDX5: DEAD box polypeptide 5; EBV: Epstein-Barr virus; CGH: Comparative Genomic Hybridization; RNU6B: U6 small nuclear 2 RNA; Ct: cycle threshold: PCYOX1L: prenylcysteine oxidase 1 like; IL17B: interleukin 17B; CSNK1A1: casein kinase 1 alpha 1; UTP: uridine triphosphate; GAPDH: glyceraldehyde-3-phosphate dehydrogenase; rRNA: ribosomal RNA;

\section{Competing interests}

The authors declare that they have no competing interests.

\section{Authors' contributions}

Al and YA conceived and planned the experiments. TN and YA provided the human cancer cell lines. YN and $\mathrm{H}$ collected the clinical specimens. Al performed all experiments. All authors read and approved the manuscript.

\section{Acknowledgements}

We thank Dr. Takagi and Dr. Tanigawa (Osaka Medical College Hospital) for supplying clinical samples. This work was supported in part by a grant-in-aid for scientific research (No. 21659104 to A.I.) from the Ministry of Education, Culture, Sports, Science, and Technology of Japan.

\section{Author Details}

1Department of Medical Oncology, Gifu International Institute of Biotechnology, 1-1 Naka-Fudogaoka, Kakamigahara, Gifu 504-0838, Japan, 2Department of Gastroenterology, Fujita Health University, School of Medicine, 1-98 Dengakugakubo, Kutsukake-cho, Toyoake, Aichi 470-1192, Japan, 3Department of Hematology and Oncology, Nagoya University, Graduate School of Medicine, 65 Tsurumai-cho, Showa-ku, Nagoya, Aichi 466-8550, Japan and 4 United Graduate School of Drug Discovery and Medical Information Sciences, Gifu University, Yanagido, Gifu, Gifu 501-1193, Japan
Received: 27 October 2009 Accepted: 2 June 2010

Published: 2 June 2010

\section{References}

1. Bartel DP: MicroRNAs: genomics, biogenesis, mechanism, and function. Cell 2004, 116:281-97.

2. Pillai RS: MicroRNA function: multiple mechanisms for a tiny RNA? RNA 2005, 11:1753-61.

3. Nilsen TW: Mechanisms of microRNA-mediated gene regulation in animal cells. Trends Genet 2007, 23:243-9.

4. Harfe BD: MicroRNAs in vertebrate development. Curr Opin Genet Dev 2005, 15:410-5.

5. Calin GA, Croce CM: MicroRNA-cancer connection: the beginning of a new tale. Cancer Res 2006, 66:7390-4.

6. Akao Y, Nakagawa Y, Naoe T: MicroRNAs 143 and 145 are possible common onco-microRNAs in human cancers. Oncol Rep 2006, 16:845-50.

7. Akao Y, Nakagawa Y, Naoe T: MicroRNA-143 and -145 in colon cancer. DNA Cell Biol.

8. Takagi T, lio A, Nakagawa Y, Naoe T, Tanigawa N, Akao Y: Decreased expression of microRNAs-143 and -145 in human gastric cancers. Oncology 2009, 77:12-21.

9. Akao $Y$, Nakagawa $Y$, Kitade $Y$, Kinoshita T, Naoe T: Down-regulation of micoRNAs-143 and -145 in B-cell malignancies. Cancer Sci 2007, 98:1914-20.

10. Lin T, Dong W, Huang J, Pan Q, Fan X, Zhang C, Huang L: MicroRNA-143 as a tumor suppressor for bladder cancer. J Urol 2009, 181:1372-80.

11. Dyrskjøt L, Ostenfeld MS, Bramsen JB, Silahtaroglu AN, Lamy P, Ramanathan R, Fristrup N, Jensen JL, Andersen CL, Zieger K, Kauppinen S, Ulhøi BP, Kjems J, Borre M, Ørntoft TF: Genomic profiling of microRNAs in bladder cancer: miR-129 is associated with poor outcome and promotes cell death in vitro. Cancer Res 2009, 69:4851-60.

12. Wang X, Tang S, Le SY, Lu R, Rader JS, Meyers C, Zheng Z-M: Aberrant expression of oncogenic and tumor-suppressive microRNAs in cervical cancer is required for cancer cell growth. PLoS One 2008, 3:e2557.

13. Michael MZ, O' Connor SM, van Holst Pellekaan NG, Young GP, James RJ: Reduced accumulation of specific microRNAs in colorectal neoplasia. Mol Cancer Res 2003, 1:882-91.

14. Slaby O, Svoboda M, Fabian P, Smerdova T, Knoflickova D, Bednarikova M, Nenutil R, Vyzula R: Altered expression of miR-21, miR-31, miR-143 and miR-145 is related to clinicopathologic features of colorectal cancer. Oncology 2007, 72:397-402.

15. Wang CJ, Zhou ZG, Wang L, Yang L, Zhou B, Gu J, Chen HY, Sun XF: Clinicopathological significance of microRNA-31, -143 and -145 expression in colorectal cancer. Dis Markers 2009, 26:27-34.

16. Motoyama K, Inoue H, Takatsuno Y, Tanaka F, Mimori K, Uetake H, Sugihara K, Mori M: Over- and under-expressed microRNAs in human colorectal cancer. Int J Oncol 2009, 34:1069-75.

17. Chen HC, Chen GH, Chen YH, Liao WL, Liu CY, Chang KP, Chang YS, Chen $\mathrm{SJ}$ : MicroRNA deregulation and pathway alterations in nasopharyngeal carcinoma. Br J Cancer 2009, 100:1002-11.

18. Tong AW, Fulgham P, Jay C, Chen P, Khalil I, Liu S, Senzer N, Eklund AC, Han J, Nemunaitis J: MicroRNA profile analysis of human prostate cancers. Cancer Gene Ther 2009, 16:206-16.

19. Akao Y, Nakagawa Y, Hirata I, lio A, Ito T, Kojima K, Nakashima R, Kitade Y, Naoe T: Role of anti-oncomirs miR-143 and -145 in human colorectal tumors. Cancer Gene Ther 2010, 17:398-408.

20. Chen X, Guo X, Zhang H, Xiang Y, Chen J, Yin Y, Cai X, Wang K, Wang G, Ba Y, Zhu L, Wang J, Yang R, Zhang Y, Ren Z, Zen K, Zhang J, Zhang CY: Role of miR-143 targeting KRAS in colorectal tumorigenesis. Oncogene 2009, 28:1385-1392.

21. Sachdeva M, Zhu S, Wu F, Wu H, Walia V, Kumar S, Elble R, Watabe K, Mo YY: p53 represses c-Myc through induction of the tumor suppressor miR-145. Proc Natl Acad Sci USA 2009, 106:3207-12.

22. Akao $Y$, Nakagawa $Y$, lio A, Naoe T: Role of microRNA-143 in Fasmediated apoptosis in human T-cell leukemia Jurkat cells. Leukemia Res 2009, 33:1530-1538.

23. Shi $B$, Sepp-Lorenzino L, Prisco M, Linsley $P$, deAngelis T, Baserga R: Micro RNA 145 targets the insulin receptor substrate- 1 and inhibits the growth of colon cancer cells. J Biol Chem 2007, 282:32582-32590. 
24. Xu N, Papagiannakopoulos T, Pan G, Thomson JA, Kosik KS: MicroRNA-145 regulates OCT4, SOX2, and KLF4 and represses pluripotency in human embryonic stem cells. Cell 2009, 137:647-58.

25. Fujita S, Iba H: Putative promoter regions of miRNA genes involved in evolutionarily conserved regulatory systems among vertebrates. Bioinformatics 2008, 24:303-8.

26. Ebisuya M, Yamamoto T, Nakajima M, Nishida E: Ripples from neighbouring transcription. Nat Cell Biol 2008, 10:1 106-13.

27. Rodriguez A, Griffiths-Jones S, Ashurst JL, Bradley A: Identification of mammalian microRNA host genes and transcription units. Genome Res 2004, 14:1902-10.

28. Suzuki HI, Yamagata K, Sugimoto K, Iwamoto T, Kato S, Miyazono K: Modulation of microRNA processing by p53. Nature 2009, 460:529-33.

29. Cordes KR, Sheehy NT, White MP, Berry EC, Morton SU, Muth AN, Lee TH, Miano JM, Ivey KN, Srivastava D: miR-145 and miR-143 regulate smooth muscle cell fate and plasticity. Nature 2009, 460:705-10.

doi: $10.1186 / 1476-4598-9-136$

Cite this article as: lio et al., Identification of non-coding RNAs embracing microRNA-143/145 cluster Molecular Cancer 2010, 9:136

Submit your next manuscript to BioMed Central and take full advantage of:

- Convenient online submission

- Thorough peer review

- No space constraints or color figure charges

- Immediate publication on acceptance

- Inclusion in PubMed, CAS, Scopus and Google Scholar

- Research which is freely available for redistribution

Submit your manuscript at www.biomedcentral.com/submit
C) Biomed Central 